\title{
EDITORIAL
}

\section{Management of hemangioblastomas in patients with von Hippel-Lindau disease: stereotactic radiosurgery compared to surgical excision}

\author{
Edward H. Oldfield, MD \\ Department of Neurological Surgery, University of Virginia Health Science Center, University of Virginia, Charlottesville, Virginia
}

$\mathrm{K}$ ANO and colleagues report the results of the analysis of a retrospective, multiinstitutional, multinational experience with stereotactic radiosurgery (SRS) for hemangioblastomas (HBs) of the brain from 19 centers. ${ }^{3}$ Their report includes treatment of $335 \mathrm{HBs}$ in 80 patients with von Hippel-Lindau disease (VHL) and 182 sporadic HBs in 106 patients.

My comments are mainly related to the patients with VHL, as all of the information on the natural history of HBs, essential information for selection of the most appropriate therapy and when to use it, is from studies of patients with VHL, since these patients are the patients with HBs who are followed with serial brain and spinal MRI.

\section{Lesions Selected for Treatment Differ Greatly Between SRS and Surgery}

It is clear from this study as well as other studies of SRS for HBs that in VHL the reports on the experience with SRS describe an entirely different set of patients compared to the surgical series; the SRS experience is almost entirely comprised of tumors that are either small HBs or are a residual of unsuccessful surgery, HBs that are visible on MRI but that would not be candidates for surgical treatment. The HBs treated by SRS are smaller, generally so small that they are not producing symptoms, and are less likely to have an associated cyst than are the HBs treated with surgery. For instance, in the Kano series of SRS the mean tumor size was $0.9 \mathrm{~cm}^{3}$ for the 335 treated tumors in the VHL patients. This compares to an average size of $2.4 \mathrm{~cm}^{3}$ in the NIH surgical series of all 164 cerebellar HBs in VHL, ${ }^{2} 4.0 \mathrm{~cm}^{3}$ in the $46 \mathrm{HBs}(28 \%)$ not associated with cysts, but $16.5 \mathrm{~cm}^{3}$ total volume in the 118 HBs $(72 \%)$ associated with cysts (so large that it is far off the scale of the graph). (See Fig. 1A.)

Kano et al. describe 48 of their 80 VHL patients treated with SRS as having symptoms. Almost all patients with
VHL with symptoms from an HB of the brain have the symptoms from one, rather than the combined effects of more than one tumor. Thus, we can assume that only 48 of the 335 SRS-treated HBs were producing symptoms (14\%). This compares to symptoms in $96 \%$ of the patients receiving surgery at the NIH (Fig. 1B), a series in which $83 \%$ of the resected HBs produced symptoms and $17 \%$ were resected as a result of being in the surgical field during surgery for a patient with a symptom-producing HB.

Cysts associated with the HB are often the largest component of the space-occupying lesion, and the great majority of symptom-producing HBs are associated with a cyst that is larger than the tumor causing it. In even greater emphasis on the great difference in the tumor size in SRStreated versus surgical patients with HBs, only $11 \%$ of the 335 tumors in VHL patients had associated cysts in the report by Kano et al.; this compares to $72 \%$ with cysts in the 164 resected HBs in the NIH series. Moreover, if the mass contributed by the cyst is included, the mean total volume of the lesions with cysts in the NIH series was $16.5 \pm 14 \mathrm{~cm}^{3}$. The difference in the percentage of treated lesions that were producing symptoms in these two series, one a large SRS series, the other a large surgical series in which patients were selected for treatment only when they had symptoms, is clearly a reflection of differences in patient selection based on symptom production dictating who was, or was not, a candidate for treatment.

Thus, in general, there are clearly substantive differences in the circumstances in which SRS and surgery have been used in patients with VHL; most of the lesions selected for surgical treatment were much larger, more likely to have associated cysts increasing the mass effect further, and were producing symptoms, whereas almost all the HBs treated with SRS have been small HBs that were detected on MRI, but were too small to produce symptoms. Despite these great differences, most of the reports of SRS for these tumors attempt to compare the 

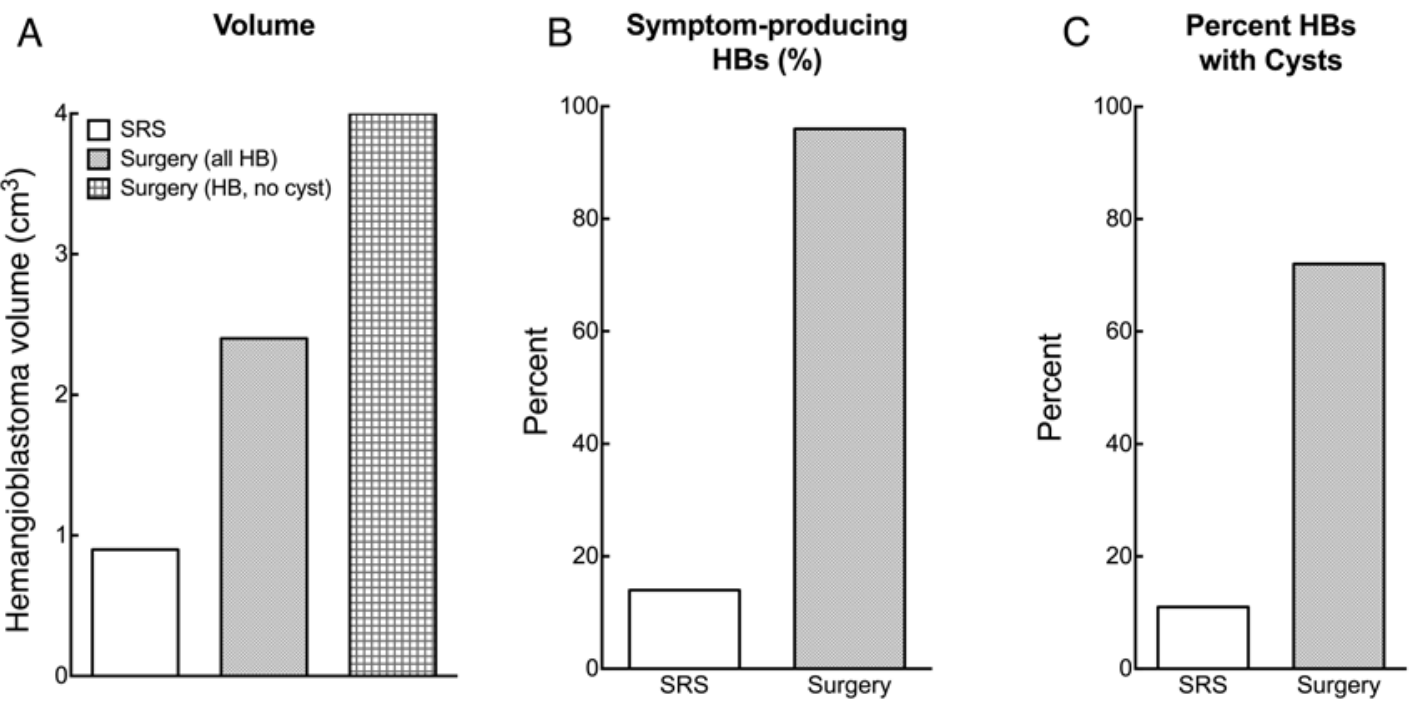

FIG. 1. These graphs show the differences in the lesions selected for treatment with SRS in the report by Kano et al. ${ }^{3}$ compared to the lesions removed surgically because they produced symptoms in the $\mathrm{NIH}$ series. ${ }^{2} \mathrm{~A}$ : The mean $\mathrm{HB}$ volume in the patients with VHL treated with SRS was substantially smaller than the volume of all solid HBs and HBs that produced symptions but were not associated with a cyst. In the surgical series, most HBs were associated with a cyst $(72 \%)$ and the average volume of the HB + cyst was $16.5 \mathrm{~cm}^{3}$, well beyond the scale of this graph. B: Substantially more HBs that received treatment were producing symptoms in the surgical series compared to those in patients receiving treatment with SRS. C: A much greater fraction of patients who received surgery had cysts associated with the HB than in the SRS series.

risks of SRS versus surgical treatment, comparisons that are clearly invalid.

\section{Risks Associated With Treatment}

Kano et al. report complications associated with SRS in 13 of the total of 186 patients treated (7\%; they do not distinguish complications between the VHL and sporadic HB patients). Eleven of the 13 patients required steroid therapy for treatment-induced edema surrounding the tumor, 1 patient died of refractory peritumoral edema, and 2 patients required surgery (one for hydrocephalous, one for peritumoral cyst drainage). In addition, 19\% of the VHL patients required additional treatment for progression of a treated tumor.

In the surgical series from the NIH, immediately after tumor resection, patients were stable or improved after $88 \%$ of operations; in $12 \%$ of operations there were new cerebellar signs and symptoms or exacerbation of preexisting symptoms. ${ }^{2}$ However, these new symptoms and signs were frequently mild in nature and resolved rapidly (mean resolution time $9.3 \pm 2.2$ days) in all but 1 case. By 3 months after surgery, patients were at their preoperative status or improved after $98 \%$ of the operations. All patients without preoperative signs or symptoms remained asymptomatic after resection. No patient died as a result of surgical treatment. Since there was complete excision of all tumors, no treated tumor required additional treatment.

\section{Optimal Management of HBs in VHL}

Is SRS the best management for small HBs in VHL that are not causing symptoms? How does the natural history of the HBs treated with SRS compare to the natural history of untreated HBs in VHL?
To establish whether SRS is a reasonable option for small HBs that are not producing symptoms in patients with VHL requires analysis of the natural history of SRStreated HBs that were not causing symptoms compared to the natural history of similar lesions that were not treated. Unfortunately, there are no randomized studies addressing this important issue. However, we can benefit from assessing the information that is available. Lonser et al. recently reported the results of a large prospective study of the natural history of 2505 untreated HBs, 1302 (52\%) of which were in the cerebellum or brainstem, in 225 patients with VHL. ${ }^{4}$ Their study included clinical and imaging assessment every 6 months for a mean of 6.9 years. Further, note that that study included untreated HBs with cysts, peritumoral edema, and new lesions. Two hundred ninetytwo new cerebellar HBs arose during the 6.9 years of the study, and $50 \%$ of the HBs in the cerebellum progressed at 6.2 years. In most lesions the growth was a stuttering, on-off, type of growth (this occurred in $72 \%$ of the lesions that enlarged), and the great majority of HBs that demonstrated growth produced no symptoms and did not require treatment. At last evaluation only 159 (6.3\%) of the 2505 HBs produced symptoms and required treatment over the mean evaluation of 6.9 years.

Of the $335 \mathrm{HBs}$ in VHL patients treated with SRS in the current study, only $14 \%$ of which were symptom-producing, and over the mean follow-up of 5.5 years, 26 (8\%) HBs progressed despite the treatment. As the authors indicate, the lesions selected for treatment either were lesions that had shown growth on serial MRI, were new lesions on MRI, or were producing symptoms. Thus, there was selection for tumors that may have had a greater likelihood of progression over time than would have been the case if all MRI-visible lesions were assessed. However, despite the 
treatment, note that $8 \%$ progressed over the 5.5 years of mean follow-up. Compare that with the $6.3 \%$ rate of progression of the untreated HBs in the NIH study-and that study included new HBs, HBs with cysts, and HBs that had shown growth.

In a prospective study, Asthagiri et al. examined the effects of SRS in 44 HBs in the cerebellum (39) and brainstem (5) in patients with VHL. ${ }^{1}$ Local control of the 44 treated HBs was only $61 \%$ at 10 years and $51 \%$ at 15 years. Once again, these were small tumors, with mean tumor volume of $0.5 \mathrm{~cm}^{3}$; despite treatment, 9 of the 27 small, asymptomatic HBs progressed (33\%) during the average of 8.5 years of follow-up.

\section{Summary}

Symptom-producing HBs need treatment. Essentially all sporadic HBs are diagnosed as a result of the symptoms that they produce. A series of studies have shown that in the hands of experienced surgeons HBs of the cerebellum and brainstem can almost always be safely and completely removed surgically with a risk of recurrence approaching zero. ${ }^{2,5}$ Most symptom-producing HBs have either an associated cyst or a region of edema surrounding them, features associated with increased risk of adverse effects and poor tumor control after SRS, as is yet again demonstrated in the study by Kano and colleagues. The authors describe an incidence of $43 \%$ for new lesions in VHL patients at 5 years and $84 \%$ at 10 years. Among the lesions selected for treatment with SRS were new lesions. ${ }^{3}$ Do $84 \%$ of patients with VHL need to have SRS every 10 years?

In patients with VHL, most HBs treated with radiosurgery have been small and asymptomatic. The incidence of progression of even small HBs treated with radiosurgery is not greatly different from their natural history if untreated. In VHL, since we cannot reliably predict which lesions will produce symptoms and require treatment, current knowledge favors introducing treatment when HBs produce symptoms and the use of surgery when that occurs.

The bottom line for a patient with an HB producing symptoms? Find a neurosurgeon with significant surgical experience with these tumors.

http://thejns.org/doi/abs/10.3171/2015.2.JNS15252

\section{References}

1. Asthagiri AR, Mehta GU, Zach L, Li X, Butman JA, Camphausen KA, et al: Prospective evaluation of radiosurgery for hemangioblastomas in von Hippel-Lindau disease. Neuro Oncol 12:80-86, 2010

2. Jagannathan J, Lonser RR, Smith R, DeVroom HL, Oldfield EH: Surgical management of cerebellar hemangioblastomas in patients with von Hippel-Lindau disease. J Neurosurg 108:210-222, 2008

3. Kano H, Shuto T, Iwai Y, Sheehan J, Yamamoto M, McBride HL, et al: Stereotactic radiosurgery for intracranial hemangioblastomas: a retrospective international outcome study. J Neurosurg [epub ahead of print March 27, 2015. DOI: 10.3171/2014.10.JNS131602]

4. Lonser RR, Butman JA, Huntoon K, Asthagiri AR, Wu T, Bakhtian KD, et al: Prospective natural history study of central nervous system hemangioblastomas in von HippelLindau disease. J Neurosurg 120:1055-1062, 2014

5. Wind JJ, Bakhtian KD, Sweet JA, Mehta GU, Thawani JP, Asthagiri AR, et al: Long-term outcome after resection of brainstem hemangioblastomas in von Hippel-Lindau disease. J Neurosurg 114:1312-1318, 2011

\section{Response}

\section{Hideyuki Kano, MD, PhD, ${ }^{1}$ Masaaki Yamamoto, MD, ${ }^{2}$ and L. Dade Lunsford, MD1}

'Department of Neurological Surgery, University of Pittsburgh, Pennsylvania; and ${ }^{2}$ Katsuta Hospital Mito GammaHouse, Hitachinaka, Japan

We appreciate the concise and clear analysis performed by Dr. Oldfield related to our paper, which describes the outcomes in a large group of patients who underwent SRS for symptomatic or progressively growing HBs. We would like to respond with a few additional points to amplify the results of our study. We acknowledge Dr. Oldfield's extensive experience with VHL-associated HBs. Stereotactic radiosurgery is a minimally invasive option specifically selected for patients with growing tumors who have tumor volumes (and without surgically accessible local mass effect) that do not, as yet, require craniotomy and surgical excision. As noted clearly in our report, such HBs tended to be smaller, be less symptomatic, and have lower rates of cystic components compared to those for which Dr. Oldfield selected surgical excision during his experience at the NIH. ${ }^{1}$ We agree that surgery should be considered as the primary option for patients with symptomatic, progressive sporadic or VHL-associated HBs-when the tumors are in surgically accessible brain regions and the surgery can be expected to entail a low risk of morbidity when performed by an experienced neurosurgeon. In the present study, most patients with VHL-associated HB who underwent SRS had long-term tumor growth control. In contrast, patients with sporadic HBs tended to have higher rates of tumor progression after SRS. We concur that most small, asymptomatic HBs detected in patients with VHL warrant observation until progressive growth or clinical symptoms emerge-a strategy appropriate for most patients with histologically "benign" brain tumors.

However, we recommend that patients with progressive tumor growth should be considered for SRS before they require a craniotomy as the only option for management. VHL patients may require multiple such procedures over the course of their lives. As in many neurosurgical conditions for which we have an increasingly high level of evidence, we can always toss out the expected platitude that only a prospective randomized controlled study comparing resection and SRS in the treatment of newly emerged VHL-associated HBs will answer our questions. Sadly, neither governments, nor insurance companies, nor industry, nor patient support groups, nor professional societies will fund such trials. In the mean time we believe that both VHL-associated and sporadic HB patients should find neurosurgical centers where experienced neurosurgeons with both microsurgical and radiosurgical skills exist.

\section{Reference}

1. Jagannathan J, Lonser RR, Smith R, DeVroom HL, Oldfield EH: Surgical management of cerebellar hemangioblastomas in patients with von Hippel-Lindau disease. J Neurosurg 108:210-222, 2008 